\title{
XB130: A novel adaptor protein in cancer signal transduction (Review)
}

\author{
RUIYAO ZHANG ${ }^{1 *}$, JINGYAO ZHANG $^{1 *}$, QIFEI WU ${ }^{2}$, FANDI MENG $^{1}$ and CHANG LIU ${ }^{1}$ \\ Departments of ${ }^{1}$ Hepatobiliary Surgery and ${ }^{2}$ Thoracic Surgery, The First Affiliated Hospital \\ of Xi'an Jiaotong University, Xi'an, Shannxi 710061, P.R. China
}

Received February 2, 2015; Accepted January 18, 2016

DOI: $10.3892 /$ br.2016.588

\begin{abstract}
Adaptor proteins are functional proteins that contain two or more protein-binding modules to link signaling proteins together, which affect cell growth and shape and have no enzymatic activity. The actin filament-associated protein (AFAP) family is an important member of the adaptor proteins, including AFAP1, AFAP1L1 and AFAP1L2/XB130. AFAP1 and AFAP1L1 share certain common characteristics and function as an actin-binding protein and a cSrc-activating protein. XB130 exhibits certain unique features in structure and function. The mRNA of XB130 is expressed in human spleen, thyroid, kidney, brain, lung, pancreas, liver, colon and stomach, and the most prominent disease associated with XB130 is cancer. XB130 has a controversial effect on cancer. Studies have shown that XB130 can promote cancer progression and downregulation of XB130-reduced growth of tumors derived from certain cell lines. A higher mRNA level of XB130 was shown to be associated with a better survival in non-small cell lung cancer. Previous studies have shown that XB130 can regulate cell growth, migration and invasion and possibly has the effect through the cAMP-cSrc-phosphoinositide 3-kinase/Akt pathway. Except for cancer, XB130 is also associated with other pathological or physiological procedures, such as airway repair and regeneration.
\end{abstract}

\section{Contents}

1. Introduction

2. Adaptor proteins: Major contributors to signaling pathways

3. AFAP family: Important adaptor protein family

Correspondence to: Professor Chang Liu, Department of Hepatobiliary Surgery, The First Affiliated Hospital of Xi'an Jiaotong University, 277 Yanta West Street, Xi'an, Shannxi 710061, P.R. China E-mail: liuchangdoctor@163.com

*Contributed equally

Key words: adaptor proteins, actin filament-associated protein, XB130, cancer
4. XB130: A newly studied adaptor protein in cancer signal transduction

5. Conclusion

\section{Introduction}

Adaptor proteins are functional proteins that contain two or more protein-binding modules to link signaling proteins together, which affect cell growth and shape and have no enzymatic activity. The actin filament-associated protein (AFAP) family is an important member of the adaptor proteins, including AFAP1, AFAP1L1 and AFAP1L2/XB130. AFAP1 and AFAP1L1 share certain common characteristics and function as an actin-binding protein and a cSrc-activating protein. XB130 exhibits certain unique features in structure and function. The mRNA of XB130 is expressed in the human spleen, thyroid, kidney, brain, lung, pancreas, liver, colon and stomach, and the most prominent disease associated with XB130 is cancer. XB130 has a controversial effect on cancer. Studies have shown XB130 could promote cancer progression and down-regulation of XB130 reduced growth of tumors derived from some cell lines. While a higher mRNA level of XB130 was shown to be associated with a better survival in non-small cell lung cancer. Research has shown that XB130 could regulate cell growth, migration and invasion and play the effect probably through cAMP-cSrc-phosphoinositide 3-kinase (PI3K)/Akt pathway. In addition to cancer, XB130 is associated with other pathological or physiological procedures, such as airway repair and regeneration.

\section{Adaptor proteins: Major contributors to signaling pathways}

Adaptor proteins are commonly defined as proteins that contain two or more protein-binding modules that function to link signaling proteins together, which affects cell growth and shape and have no enzymatic activity (1). As the response of cells to external stimuli shows specificity and veracity, these adaptor proteins have received increasing attention when attempting to elucidate the mechanisms that control the cross-talk between signaling cascades (2). The varieties of protein-binding modules enable adaptor proteins to link protein-binding partners together and thus create larger 
signaling complexes, leading to an appropriate response from the cell to the environment (3). The specificity in signaling can be achieved by the type of protein-binding modules, the sequence of these domains and motifs encoded by adaptor proteins, the subcellular locations and the proximity of binding partners $(1,3)$. The specificity and veracity of adaptor proteins provide the cell with a mechanism to link signaling proteins to each other and propagate a cellular signal.

A large number of studies regarding adaptor proteins have been carried out since the earlier definition of functional domains within proteins and the subsequent identification of their function. The function of the Src homology 2 (SH2) domain was first demonstrated, and later, a number of other protein-binding modules such as $\mathrm{SH}$, pleckstrin homology (PH), WW and other domains, as well as the conservative structure of the opposing binding motifs were identified (4-7). Furthermore, studies established that the integrity of these protein-binding modules was functionally required for $\mathrm{v}-\mathrm{Src}$ or Src527F to transform cells, while mutations of these $\mathrm{SH} 2$ or SH3 domains of $\mathrm{v}$-Src or Src527F could prevent these oncogene products from transforming cells $(8,9)$. It is the structural analysis of these modular domains using X-ray crystallography or nuclear magnetic resonance (NMR) that finally demonstrated the active centers of these domains and the mechanisms by which they facilitate protein-protein interactions, leading to the acknowledgement of the importance of protein-protein interactions in modulating cellular signaling (10-13).

The former functional structure of adaptor proteins that are limited in SH2 and SH3-binding sites were broadened by the identification of Crk $(14,15)$. Since then, a large number of members of adaptor proteins have been recognized and studied, and it has been demonstrated that adaptor proteins are involved in multiple signaling pathways through different up and downstream molecules. The T-cell receptor signaling adaptor proteins, LAT, GADS and SLP-76, promote receptor-regulated signaling, as examples that modulate signals emanating from the cell membrane (16-19). It was also found that the former mentioned Crk family has an important role in modulating cell adhesion and cell migration (20). Furthermore, the Vav family was highlighted as co-evolved with tyrosine kinases, as a mechanism to link changes in the cytoskeletal architecture and gene transcription $(21,22)$. Continuing the theme of adaptor proteins and the cytoskeleton, several tyrosine phosphorylated substrates, including pp130cas, pp125FAK, AFAP-110, pp85 cortactin and the cadherin-associated protein pp120ctn, have been identified (23-26). Notably, each of these tyrosine-phosphorylated substrates is associated with the actin-based cytoskeleton, and AFAP-110 has been proved to be capable of regulating actin filament integrity as an activator of Src (25).

Significantly, there have been studies that are engaged in investigating the target rational drug design to block the ability of certain adaptor proteins to form interactions with cellular signaling proteins. These studies attempted to design certain small molecular inhibitors that specifically block functional interactions forged by adaptor proteins $(8,27-31)$. An improved understanding of adaptor proteins in signal transduction is required prior to their use as potential targets for therapy.

\section{AFAP family: Important adaptor protein family}

The AFAP family, which is an important member of the adaptor proteins, consists of three members: AFAP1, actin filament-associated protein 1-like 1 (AFAP1L1), and AFAP1L2 (also known as XB130), among which the prototype member, AFAP1, also termed AFAP-110, is well acknowledged as a cSrc-binding partner and actin filament cross-linking protein $(32,33)$. AFAP1, AFAP1L1 and AFAP1L2 (XB130) are classified as a family by the Human Genome Project for their similar modular domain structure and amino acid sequence, particularly for their highly conservative PH domains $(34,35)$.

AFAP1 can be used as an example to introduce the typical structure of this AFAP family. The AFAP1 protein contains two juxtaposed poly-proline rich SH3-binding motifs, two SH2-binding motifs, two PH domains, a substrate domain (SD), a helical leucine zipper (Lzip) for multimerization and an actin-binding domain, which is used for AFAP1 to interact with actin filaments. A comparative study between AFAP1 and AFAP1L1 shows $71 \%$ similarity in the overall amino acid structure between the two proteins, and specifically, AFAP1L1 only contains one putative SH3-binding motif, and this motif more closely resembles that of a cortactin SH3-domain binding site, compared with the cSrc SH3-binding motif of AFAP1 (36).

As mentioned previously, AFAP1 is known to function as an actin-binding protein and a cSrc-activating protein (33). The carboxy terminal actin filament-binding domain and the Lzip of AFAP1 enable it to crosslink actin filaments (37). Following activation by the serine/threonine kinase PKC $\alpha$, AFAP1 is directed to perinuclear region of the cell with cSrc and activates cSrc by binding to the $\mathrm{SH} 3$ domain via its N-terminal SH3-binding motif, thus leading to the cSrc downstream activation that affects cell adhesion, invasion and motility (25). Similar to AFAP1, AFAP1L1 also has an ability to associate with actin filaments, can be found in actin-rich structures such as invadosomes, and is capable of independently inducing podosomes upon overexpression (38). Additionally, AFAP1L1 exhibits certain unique characteristics. For example, AFAP1L1 was identified in the dentate nucleus and its expression appeared to occur along neuronal processes, which indicates its unique role in the innervation of the dentate nucleus (39).

However, as opposed to AFAP1 and AFAP1L1 showing a number of common features, the third member, AFAP1L2 (XB130), exhibits differences in structure and function. This is further discussed in the following.

\section{XB130: A newly studied adaptor protein in cancer sig- nal transduction}

Location and distribution of XB130. A novel protein emerged when investigators searched for the human AFAP gene. As this protein does not show any evident enzymatic domain, but contains certain modules for protein-protein interaction, and shows a distinguished structure similar to AFAP, it was designated as AFAP1L2. In addition, AFAP1L2 encodes a protein of 818 amino acids and the molecular mass measured by immunoblotting is $\sim 130 \mathrm{kDa}$. Therefore, it is also, and more commonly, known as XB130 (35). 
Fluorescence in situ hybridization analysis shows that the XB130 gene is localized on human chromosome 10q25.3, and this location is further confirmed by a GenBank ${ }^{\mathrm{TM}}$ database search (35). Although XB130 has not attracted significant attention until recent years, it has been established that XB130 is a comparatively widely expressed protein. A previous study identified that the mRNA is frequently expressed in human spleen and thyroid, and although it si relatively lower, its expression could also be detected in the kidney, brain, lung, pancreas, liver, colon and stomach (40). Additionally, different approaches using several cell lines showed that XB130 tends to be distributed in the cytoplasm.

Structure of XB130. A total of 818 amino acids form the primary structure of XB130. In general, XB130 contains several SH2- and SH3-binding motifs, two PH domains, a coiled-coil region, and a number of potential tyrosine or serine/threonine phosphorylation sites (35). Specifically, sequence structure analysis shows that the N-terminal region of XB130 contains 23 putative tyrosine phosphorylation sites and one proline-rich motif that may interact with $\mathrm{SH} 2$ - and $\mathrm{SH} 3$-domain-containing proteins, respectively (41). Subsequently, they are followed by two PH domains that target host proteins to cellular membranes through interactions with certain phospholipids and membrane-associated proteins $(42,43)$, and its C-termini shows a coiled-coil domain, partially similar with the Lzip domain in AFAP. The coiled-coil and Lzip domains are believed to be involved in protein oligomerization and DNA binding $(44,45)$.

XB130 in cancer. Recently, XB130 has attracted increasing interest among researchers to a significant extent owing to its controversial effect on cancers. Pathway analysis showed that the top-ranked disease associated with XB130 is cancer (46). A study associating XB130 and prognosis in non-small cell lung cancer showed that the overall expression of XB130 in adenocarcinoma (ADC) was much lower than that in squamous cell carcinoma (SQCC), and a higher XB130 mRNA level independently indicated an improved disease-free survival at 5 years. This provided evidence that XB130 could be a marker to distinguish ADC from SQCC and to predict a long-term survival in non-small cell lung cancer patients (47). The same result was obtained in an in vitro study, as the downregulation of XB130 reduced growth of tumors derived from A549 cells, a human lung carcinoma cell line. A similar result was also shown in A549 cells, a human lung carcinoma cell line (35).

Several studies concerning the interaction between XB130 and Src kinases, oncogenic RET/PTC and other signaling molecules have identified its involvement in tumor progression by promoting cell proliferation and survival in various tumor cells. Shiozaki et al (48) found that XB130 was highly expressed in human thyroid cancers. The in vitro study by Lodyga et al (49) of the TPC1 cell line identified that downregulation of XB130 in the TPC1 cell line derived from papillary thyroid carcinoma led to proliferation reduction and anoikis promotion. In addition, downregulation of XB130 reduced the growth of tumors derived from WRO cells (41). Knockdown of XB130 in WRO cells inhibited the G1-S phase progression, induced spontaneous apoptosis and enhanced intrinsic and extrinsic apoptotic stimulus-induced cell death. Microarray analysis identified that 246 genes were significantly changed in XB130 small hairpin RNA (shRNA)-transfected cells, among which 57 genes were involved in cell proliferation and survival, as well as transcriptional regulation. To further explore the mechanisms that XB130 regulates gene expression associated with cell proliferation or survival, Takeshita et al (50) analyzed miRNA expression in XB130 knockdown WRO cells, based on the estimation that $30 \%$ of all human genes can be regulated by miRNAs (51). They speculated that XB130 may regulate growth-related expression of miRNAs and in this way control the genes associated with cell proliferation or survival. An miRNA array assay showed that 16 miRNAs were upregulated and 22 miRNAs were downregulated significantly in WRO thyroid cancer cells with XB130 downregulation by shRNA. Furthermore, overexpression of XB130 suppressed 3 of the upregulated miRNAs (miR-33a, miR-149 and miR-193a-3p), which would negatively regulate oncogenes expression when transfected into WRO cells. In this way, investigators concluded that XB130 could promote cancer cell growth by regulating tumor-related miRNAs and their targeted genes, and this regulatory effect was inhibited by the deletion of the N-terminus of XB130, suggesting the regulation of these miRNAs by XB130 may be mediated through specific signal transduction pathways.

Investigators have also established that XB130 could regulate cell migration and invasion through its impact on cytoskeletal functions. Lodyga et al (41) showed that in MTLn3 rat mammary carcinoma cells and in natural XB130 expressors, such as a thyroid papillary carcinoma cell line TPC1, a variety of stimuli [epidermal growth factor (EGF), phorbol myristate acetate and cell wounding] induced translocation of endogenous or heterologously expressed XB130 from the cytosol to the cell periphery, where it colocalized with the simultaneously formed, membrane-associated actin meshwork. This actin meshwork assembles densely and dynamically under the plasma membrane, exerting a force upon the plasma membrane, giving rise to the formation of lamellipodia and membrane ruffles. Lamellipodia are essential for the formation of migratory membrane protrusions, an event that is closely associated with the epithelial-mesenchymal transition (EMT) (52). Specifically, it is the RAC GTPase that induces the XB130 in the lamellipodia. Lodyga et al (41) also reported that not only XB130 has the special affinity to the branched F-actin, but also the formation of the actin meshwork could importantly recruit XB130 to this site (41). A systematic mutational analysis further revealed that the XB130 N-terminus (167 amino acids) and C-terminus (63 amino acids) harbor crucial regions for its translocation to lamellipodia, whereas the PH domains and Src-targeted tyrosines are dispensable. This cytoskeleton-associated function of XB130 leads to its significance in cell migration and invasion. Knockdown of XB130 in thyroid papillary carcinoma cell lines resulted in slower cell migration in a wound-healing model and diminished matrigel invasion, indicating that XB130 impacts key cytoskeletal functions and an underlying mechanism accounting for impaired migration and invasion (41). The interaction between XB130 and Src and PI3K may explain the more specific mechanisms of its impact on cell migration and invasion. Src kinases are well-known regulators of cell migration and invasion, and PI3K and the downstream Akt are known to promote migration and 
invasion. The translocation of XB130 helps to localize Src and Akt to the sub-cytomembrane branched F-actin region, which further contributes to the restricted, augmented and prolonged activities of Src and PI3K. In this way, XB130 may have a role in modifying tumor cell motility and invasion.

The association between XB130 and tumors is explored in other types of cancer as well. For example, a multivariate analysis of biomarkers and clinical features in postoperative hepatocellular carcinoma (HCC) carried out by Zuo et al (53) showed that the positive expression rates of the XB130 protein in Chinese patients with HCC is high (75.0\%), but its expression level is not associated with postoperative prognosis $(\mathrm{P}=0.848)$. Shiozaki et al (54) explored the possible role of XB130 in cell cycle progression of esophageal squamous cell carcinoma (ESCC) cells and its expression and effects on the prognosis of patients with ESCC. In the study, XB130 was found expressed in precancerous tissue such as severe dysplasia, suggesting that XB130 may be induced in ESCC cells at an early stage, indicating its potential as a biomarker of squamous intraepithelial neoplasia of the esophagus. Furthermore, the study reported that XB130 expression in five ESCC cell lines, and knockdown of XB130 with siRNA reduced the G1-S phase in cell cycle progression in all these cell lines. Additionally, downregulation of XB130 led to an increase in expression of p21, which is also involved in cell cycle regulation. The similar effect on p21 was also observed in A549 cells, a human lung carcinoma cell line. Furthermore, the immunohistochemistry of human ESCC samples in the study revealed that expression of XB130 in cancer tissue was observed in $71.2 \%$ of patients. Of note, this study established XB130 expression in the nucleus as an independent poor prognostic factor for patients with ESCC. The 5-year survival rate of patients without nuclear XB130 expression was $81.5 \%$, significantly higher than those with nuclear XB130 expression (56.0\%). By contrast, cytoplasmic XB130 expression did not show a similar result (54).

Furthermore, a retrospective study reviewing 76 consecutive patients with surgically resected pancreatic ductal adenocarcinoma (PDAC) was carried out to clarify the prognostic significance of XB130 expression in PDAC (55). The study reported that XB130 expression in PDAC was significantly enhanced (43/76) compared to the normal pancreas $(0 / 15)$. Increased XB130 expression was correlated with lymph node metastasis, distant metastasis, high tumor-node-metastasis stage, and high-tumor grade, resulting in a worse survival than that of the patients with lower expressed XB130. Furthermore, high XB130 expression was also identified as an independent prognostic factor of postoperative survival and an independent risk factor.

However, in contrast to the studies indicating a cancer promotion effect of XB130, Shi et al (40) found evidence suggesting that the XB130 mRNA and protein were constitutively expressed in normal gastric tissue, while relatively lower expressed in gastric cancer (GC) cells (40). The study analyzed survival or recurrence in 411 GC patients and noted that low XB130 expression predicted a lower survival and higher recurrence. Shi et al explained doubt with regards to their result, as according to previous studies, XB130 should be more regarded as an oncogenic instead of a tumor-suppressive protein.

Furthermore, Shi et al (52) carried out a second study, in which they concluded that XB130 contributes to GC cell proliferation and invasiveness and is involved in phosphorylation of Akt and EMT-like changes. In this study, the proliferation, migration and invasion of two types of gastric adenocarcinoma cell lines were significantly inhibited by XB130 knockdown using shRNA, and following XB130 knockdown, tumor growth was inhibited and GC cells showed a more epithelial-like phenotype, indicating XB130 has an effect on inhibition of the EMT process. Additionally, XB130 knockdown led to the reduction of p-Akt/Akt expression, upregulated expression of epithelial markers including E-cadherin, $\alpha$-catenin and $\beta$-catenin, downregulated mesenchymal markers including fibronectin and vimentin, and reduction of oncoproteins expression associated with tumor metastasis, such as matrix metalloproteinase 2 (MMP2), MMP9, and cluster of differentiation 44.

Mechanism of XB130 in cancer. As a member of adaptor proteins, XB130 links signaling components to form multi-molecular complexes to induce and pass on cellular signals through its modular domains. Biological events are thus triggered depending on these multi-molecular interactions. Therefore, XB130, similar to other adaptor proteins, has an important role in different signaling pathways. c-Src protein-tyrosine kinases (PTKs) can be used as an example. Of note, the Src protein was the first tyrosine kinase to be discovered. Activation of c-Src leads to the downstream signal propagation such as post-translational modification of effectors, transcriptional activation of target genes and DNA synthesis $(56,57)$. c-Src has crucial roles in signaling involved in mitogenesis, differentiation, inflammation, cell survival, motility and adhesion (58-60). The common structure of Src PTKs, including c-Src, contains an N-terminal myristoylation sequence, SH3 and SH2 domains, a kinase domain, and a $\mathrm{C}$-terminal regulatory tail (61). The $\mathrm{N}$-terminal region functions to associate c-Src with the cell membrane (35). C-Src kinases are usually kept off by an autoinhibitory mechanism based on its SH2 domain and two major phosphorylation sites: Tyr416 (or Y416) and Tyr527. Tyr416 can be auto-phosphorylated, which activates Src by displacing the P-Tyr416 from the binding pocket, allowing the substrate to gain access. Phosphorylation of Tyr527 inactivates Src through the interaction of P-Tyr527 with the SH2 domain, which effectively folds Src up into a closed, inhibitory molecule. Tyr527 could be dephosphorylated directly by protein-tyrosine phosphatases or by moving Src to sites of action through binding partner, leading to the activation of Src tyrosine kinase (62-64).

$\mathrm{Xu}$ et al (35) reported that XB130 activated c-Src when they were co-expressed in COS-7 cells, and recombinant XB130 directly activated c-Src in vitro. With GST-fusion proteins, the study demonstrated that XB130 binds Src SH2 and $\mathrm{SH} 3$ sites, which explains why c-Src binding, activation and c-Src-mediated SRE transcriptional activation were all reduced when deleting the $\mathrm{N}$-terminal $\mathrm{SH} 3$ - and $\mathrm{SH} 2$-binding sites of XB130. Studies have identified that adaptor proteins would be tyrosine phosphorylated when their polyproline motif interacted with $\mathrm{Src} \mathrm{SH} 3$ domain to form additional binding sites for the Src SH2 domain. Therefore, XB130 could function as an activator and substrate of c-Src $(65,66)$.

Furthermore, $\mathrm{Xu}$ et al (35) found that upon interaction of XB130 and c-Src, AP-1 and SRE were transactivated 
depending on the level of XB130 expression. Additionally, this interaction could also increase transcriptional activities of EGF-induced SRE and interleukin-8 (IL-8) promoter. By reducing the level of XB130, certain cell lines showed a reduction of IL-8 production and a partial reduction of EGF-induced phosphorylation of Akt and glycogen synthase kinase $3 \beta$ (GSK3 $\beta$ ). IL-8 is an important inflammatory cytokine and GSK-3 activity has been reported to suppress cell proliferation. In this way, Xu et al (35) speculated that XB130 may function in inflammatory and cell cycle progression. Although the signaling mechanism of this effect was not fully explored in their study, the result showed that the interaction of XB130 and c-Src could be physiologically regulated based on the unique effect on transcriptional activities.

In addition to the role as an activator and a substrate of $\mathrm{Src}$ tyrosine kinases, XB130 is also found to be largely expressed in thyroid follicular cells, in which it serves as a prime target for the oncogenic tyrosine kinase RET/PTC, a genetically rearranged, constitutively active, thyroid-cancer-specific tyrosine kinase and the pathogenic factor in papillary carcinoma (49). In this signaling, XB130 is phosphorylated through RET/PTC. p-XB130 subsequently recruits and activates PI3K, which in turn stimulates the Akt pathway, further leading to cell proliferation and enhanced abilities of cell migration and invasion.

However, XB130 was also identified in different cancer cells in the absence of RET/PTC, and subsequent to stimulating Akt, XB130 was found to regulate cancer cell cycle progression and survival through multiple Akt downstream molecules (67). For example, the cyclin-dependent kinase inhibitor p27Kip1 nuclear translocation can cause G1 arrest and the cyclin-dependent kinase inhibitor p21Cip1/WAF1 can negatively modulate cell cycle progression by inhibiting the activation of the cyclin/cdk2 complex. The activated Akt is able to phosphorylate p27Kip1, blocking its translocation and function, and is able to phosphorylate p21Cip1/WAF1 and keep it in the cytoplasm $(68,69)$. Knockdown of XB130 with siRNA treatment reduced phosphorylation of p27Kip1 and p21Cip1/WAF1. In addition, other molecules such as FOXO3a, GSK3b, caspase 8 and caspase 9 were also identified to be the downstream molecules of Akt regulated by XB130. As Akt has been suggested as a crucial role in regulating gene transcription, cell cycle progression, and survival, implicated in a number of types of cancer $(70,71)$, XB130 could be a potential therapeutic target as the upstream regulator of PI3K/Akt pathway.

In rat FRTL-5 thyroid cells, Yamanaka et al (72) found that pretreatment of TSH or cAMP potentiated insulin-like growth factor-I-dependent DNA synthesis, and XB130 knockdown significantly decreased this cAMP-dependent effect. cAMP increased tyrosine phosphorylation of a $125-\mathrm{kDa}$ protein (p125), a rat ortholog of human XB130, and also increased its interaction with a $\mathrm{p} 85$ subunit of PI3K, which has been suggested to regulate potentiation of DNA synthesis. Furthermore, the study established that cAMP could activate cSrc, enhance XB130 mRNA and protein levels, as well as elevate XB130 phosphorylation associated with cSrc and its interaction with $\mathrm{p} 85 \mathrm{PI} 3 \mathrm{~K}$, leading to PI3K activation, associating XB130 with DNA synthesis potentiation.

XB130 in other related diseases. As previously mentioned, XB130 expression could be detected in various normal tissues of human including spleen, thyroid, kidney, lung, brain and pancreas (54). The expression of XB130 was detected in normal esophageal glands and in the cytoplasm of thyroid follicular cells in normal thyroid tissue, and no expression was identified in the normal epithelium $(48,49,54)$. These findings indicate that XB130 may have an important role in normal glandular apparatus. Aside from cancer, XB130 is also found as involved in other pathological or physiological procedures. For example, XB130 may have a role in airway epithelium differentiation during the airway repair and regeneration. In a mouse isogenic tracheal transplantation model, cells with different morphology were observed in XB130 knockout mice, which indicates different differentiation status of airway epithelial cells (73).

\section{Conclusion}

The organ-specific expression of XB130 has been identified along with its effect on cell proliferation or survival and cell motility and morphology, leading to its significant role in physiological and pathological procedures, particularly in cancer initiation and progression. In numerous types of cancer, XB130 showed a pro-tumor effect and tended to indicate a poor prognosis. With the further and comprehensive understanding of its effect and the mechanisms, XB130 could be of great importance in clinical work, including the diagnosis of precancerous lesions, the prediction of risk and prognosis, and most importantly, the target treatment in cancer. As a tumor-encouraging factor, XB130 has its effect as the upstream molecule of PI3K/Akt signaling pathway and as the upregulator of a number of tumor-related miRNAs. By targeting XB130 with its specific antibody, the multiple downstream signaling ways of Akt and tumor-stimulating genes would be silenced and tumor-suppressive genes would be uninhibited. In this way tumor progression could be blocked.

\section{References}

1. Flynn DC: Adaptor proteins. Oncogene 20: 6270-6272, 2001.

2. Pawson T and Scott JD: Signaling through scaffold, anchoring and adaptor proteins. Science 278: 2075-2080, 1997.

3. Tsyba L, Nikolaienko O, Dergai O, Dergai M, Novokhatska O, Skrypkina I and Rynditch A: Intersectin multidomain adaptor proteins: Regulation of functional diversity. Gene 473: 67-75, 2011.

4. Koch CA, Anderson D, Moran MF, Ellis C and Pawson T: SH2 and SH3 domains: Elements that control interactions of cytoplasmic signaling proteins. Science 252: 668-674, 1991.

5. Cantley LC: The phosphoinositide 3-kinase pathway. Science 296: 1655-1657, 2002.

6. Dong H, O'Brien RJ, Fung ET, Lanahan AA, Worley PF and Huganir RL: GRIP: A synaptic PDZ domain-containing protein that interacts with AMPA receptors. Nature 386: 279-284, 1997.

7. Denlinger LC, Fisette PL, Sommer JA, Watters JJ, Prabhu U, Dubyak GR, Proctor RA and Bertics PJ: Cutting edge: The nucleotide receptor $\mathrm{P} 2 \mathrm{X} 7$ contains multiple protein-and lipid-interaction motifs including a potential binding site for bacterial lipopolysaccharide. J Immunol 167: 1871-1876, 2001.

8. Schlaepfer DD, Hanks SK, Hunter T and van der Geer P: Integrin-mediated signal transduction linked to Ras pathway by GRB2 binding to focal adhesion kinase. Nature 372: 786-791, 1994.

9. Kurokawa K, Mochizuki N, Ohba Y, Mizuno H, Miyawaki A and Matsuda M: A pair of fluorescent resonance energy transfer-based probes for tyrosine phosphorylation of the CrkII adaptor protein in vivo. J Biol Chem 276: 31305-31310, 2001.

10. Musacchio A, Smith CJ, Roseman AM, Harrison SC, Kirchhausen $\mathrm{T}$ and Pearse BM: Functional organization of clathrin in coats: Combining electron cryomicroscopy and X-ray crystallography. Mol Cell 3: 761-770, 1999. 
11. Zuiderweg ER: Mapping protein-protein interactions in solution by NMR spectroscopy. Biochemistry 41: 1-7, 2002.

12. Rosen MK, Yamazaki T, Gish GD, Kay CM, Pawson T and Kay LE: Direct demonstration of an intramolecular SH2-phosphotyrosine interaction in the Crk protein. Nature 374: 477-479, 1995.

13. Dyson HJ and Wright PE: Unfolded proteins and protein folding studied by. Chem Rev 104: 3607-3622, 2004.

14. Ren R, Ye ZS and Baltimore D: Abl protein-tyrosine kinase selects the Crk adapter as a substrate using SH3-binding sites. Genes Dev 8: 783-795, 1994.

15. Feller SM: Crk family adaptors-signalling complex formation and biological roles. Oncogene 20: 6348-6371, 2001.

16. Akira S, Takeda K and Kaisho T: Toll-like receptors: Critical proteins linking innate and acquired immunity. Nat Immunol 2: 675-680, 2001.

17. Liu SK, Fang N, Koretzky GA and McGlade CJ: The hematopoietic-specific adaptor protein gads functions in T-cell signaling via interactions with the SLP-76 and LAT adaptors. Curr Biol 9: 67-75, 1999.

18. Liu SK and McGlade CJ: Gads is a novel $\mathrm{SH} 2$ and $\mathrm{SH} 3$ domain-containing adaptor protein that binds to tyrosine-phosphorylated Shc. Oncogene 17: 3073-3082, 1998.

19. Clements JL, Yang B, Ross-Barta SE, Eliason SL, Hrstka RF, Williamson RA and Koretzky GA: Requirement for the leukocyte-specific adapter protein SLP-76 for normal T cell development. Science 281: 416-419, 1998.

20. Parsons JT: Focal adhesion kinase: The first ten years. J Cell Sci 116: 1409-1416, 2003

21. Bustelo XR: Vav proteins, adaptors and cell signaling. Oncogene 20: 6372-6381, 2001.

22. Tybulewicz VL: Vav-family proteins in T-cell signalling. Current Opin Immunol 17: 267-274, 2005.

23. Defilippi P, Di Stefano P and Cabodi S: P130Cas: A versatile scaffold in signaling networks. Trends Cell Biol 16: 257-263, 2006

24. Luttrell LM, Daaka Y and Lefkowitz RJ: Regulation of tyrosine kinase cascades by G-protein-coupled receptors. Curr Opin Cel Biol 11: 177-183, 1999.

25. Gatesman A, Walker VG, Baisden JM, Weed SA and Flynn DC: Protein kinase Calpha activates c-Src and induces podosome formation via AFAP-110. Mol Cell Biol 24: 7578-7597, 2004.

26. Durieu-Trautmann O, Chaverot N, Cazaubon S, Strosberg AD and Couraud P: Intercellular adhesion molecule 1 activation induces tyrosine phosphorylation of the cytoskeleton-associated protein cortactin in brain microvessel endothelial cells. J Biol Chem 269: 12536-12540, 1994.

27. Tanaka M, Gupta R and Mayer BJ: Differential inhibition of signaling pathways by dominant-negative $\mathrm{SH} 2 / \mathrm{SH} 3$ adapter proteins. Mol Cell Biol 15: 6829-6837, 1995.

28. Taganov KD, Boldin MP, Chang KJ and Baltimore D: NF-kappaB-dependent induction of microRNA miR-146, an inhibitor targeted to signaling proteins of innate immune responses. Proc Natl Acad Sci USA 103: 12481-12486, 2006.

29. Unterholzner L, Sumner RP, Baran M, Ren H, Mansur DS, Bourke NM, Randow F, Smith GL and Bowie AG: Vaccinia virus protein C6 is a virulence factor that binds TBK-1 adaptor proteins and inhibits activation of IRF3 and IRF7. PLoS Pathog 7: e1002247, 2011

30. Dawson MA, Prinjha RK, Dittmann A, Giotopoulos G, Bantscheff M, Chan WI, Robson SC, Chung CW, Hopf C, Savitski MM, et al: Inhibition of BET recruitment to chromatin as an effective treatment for MLL-fusion leukaemia. Nature 478: 529-533, 2011.

31. Zheng Y, Zhang C, Croucher DR, Soliman MA, St-Denis N, Pasculescu A, Taylor L, Tate SA, Hardy WR, Colwill K, et al: Temporal regulation of EGF signalling networks by the scaffold protein Shc1. Nature 499: 166-171, 2013.

32. Flynn DC, Leu TH, Reynolds AB and Parsons JT: Identification and sequence analysis of cDNAs encoding a 110-kilodalton actin filament-associated pp60src substrate. Mol Cell Biol 13: 7892-7900, 1993.

33. Qian Y, Baisden JM, Zot HG, Van Winkle WB and Flynn DC: The carboxy terminus of AFAP-110 modulates direct interactions with actin filaments and regulates its ability to alter actin filament integrity and induce lamellipodia formation. Exp Cell Res 255: 102-113, 2000

34. Baisden JM, Qian Y, Zot HM and Flynn DC: The actin filament-associated protein AFAP-110 is an adaptor protein that modulates changes in actin filament integrity. Oncogene 20: 6435-6447, 2001.
35. Xu J, Bai XH, Lodyga M, Han B, Xiao H, Keshavjee S, Hu J, Zhang H, Yang BB and Liu M: XB130, a novel adaptor protein for signal transduction. J Biol Chem 282: 16401-16412, 2007.

36. Snyder BN, Cho Y, Qian Y, Coad JE, Flynn DC and Cunnick JM: AFAP1L1 is a novel adaptor protein of the AFAP family that interacts with cortactin and localizes to invadosomes. Eur J Cell Biol 90: 376-389, 2011.

37. Qian Y, Gatesman AS, Baisden JM, Zot HG, Cherezova L, Qazi I, Mazloum N, Lee MY, Guappone-Koay A and Flynn DC: Analysis of the role of the leucine zipper motif in regulating the ability of AFAP-110 to alter actin filament integrity. J Cell Biochem 91: 602-620, 2004.

38. Saltel F, Daubon T, Juin A, Ganuza IE, Veillat V and Génot E: Invadosomes: Intriguing structures with promise. Eur J Cell Biol 90: 100-107, 2011.

39. Proszynski TJ, Gingras J, Valdez G, Krzewski K and Sanes JR: Podosomes are present in a postsynaptic apparatus and participate in its maturation. Proc Natl Acad Sci USA 106: 18373-18378, 2009.

40. Shi M, Huang W, Lin L, Zheng D, Zuo Q, Wang L, Wang N, Wu Y, Liao Y and Liao W: Silencing of XB130 is associated with both the prognosis and chemosensitivity of gastric cancer. PloS One 7: e41660, 2012.

41. Lodyga M, Bai X-h, Kapus A and Liu M: Adaptor protein $\mathrm{XB} 130$ is a Rac-controlled component of lamellipodia that regulates cell motility and invasion. J Cell Sci 123: 4156-4169, 2010.

42. Ma AD, Brass LF and Abrams CS: Pleckstrin associates with plasma membranes and induces the formation of membrane projections: Requirements for phosphorylation and the NH2-terminal PH domain. J Cell Biol 136: 1071-1079, 1997.

43. Wang T, Pentyala S, Rebecchi MJ and Scarlata S: Differential association of the pleckstrin homology domains of phospholipases C-beta 1, C-beta 2 and C-delta 1 with lipid bilayers and the beta gamma subunits of heterotrimeric $\mathrm{G}$ proteins. Biochemistry 38: 1517-1524, 1999.

44. Gillingham AK and Munro S: Long coiled-coil proteins and membrane traffic. Biochim Biophys Acta 1641: 71-85, 2003.

45. Zhang T, Kruys V, Huez G and Gueydan C: AU-rich element-mediated translational control: Complexity and multiple activities of trans-activating factors. Biochem Soc Trans 30: 952-958, 2002

46. Shiozaki A and Liu M: Roles of XB130, a novel adaptor protein, in cancer. J Clin Bioinforma 1: 10, 2011

47. Lodyga M, Xhi C, Anraku M, et al: P-080 Prognostic expression of a novel adaptor protein XB130 innon-small cell lung cancer. Lung Cancer 49: S135, 2005.

48. Shiozaki A, Lodyga M, Bai XH, Nadesalingam J, Oyaizu T, Winer D, Asa SL, Keshavjee S and Liu M: XB130, a novel adaptor protein, promotes thyroid tumor growth. Am J Pathol 178: 391-401, 2011.

49. Lodyga M, De Falco V, Bai X, Kapus A, Melillo RM, Santoro M and Liu M: XB130, a tissue-specific adaptor protein that couples the RET/PTC oncogenic kinase to PI 3-kinase pathway. Oncogene 28: 937-949, 2009 .

50. Takeshita H, Shiozaki A, Bai XH, Iitaka D, Kim H, Yang BB, Keshavjee S and Liu M: XB130, a new adaptor protein, regulates expression of tumor suppressive microRNAs in cancer cells. PloS One 8: e59057, 2013.

51. Yu Z, Jian Z, Shen SH, Purisima E and Wang E: Global analysis of microRNA target gene expression reveals that miRNA targets are lower expressed in mature mouse and Drosophila tissues than in the embryos. Nucleic Acids Res 35: 152-164, 2007.

52. Shi M, Zheng D, Sun L, Wang L, Lin L, Wu Y, Zhou M, Liao W, Liao Y, Zuo Q and Liao W: XB130 promotes proliferation and invasion of gastric cancer cells. J Transl Med 12: 1, 2014.

53. Zuo Q, Huang H, Shi M, Zhang F, Sun J, Bin J, Liao Y and Liao W: Multivariate analysis of several molecular markers and clinicopathological features in postoperative prognosis of hepatocellular carcinoma. Anat Rec (Hoboken) 295: 423-431, 2012.

54. Shiozaki A, Kosuga T, Ichikawa D, Komatsu S, Fujiwara H, Okamoto K, Iitaka D, Nakashima S, Shimizu H, Ishimoto T, et al: $\mathrm{XB130}$ as an independent prognostic factor in human esophageal squamous cell carcinoma. Ann Surg Oncol 20: 3140-3150, 2013.

55. Zhang J, Jiang X and Zhang J: Prognostic significance of XB130 expression in surgically resected pancreatic ductal adenocarcinoma. World J Surg Oncol 12: 49, 2014.

56. Courtneidge S: Role of Src in signal transduction pathways. The Jubilee Lecture. Biochem Soc Trans 30: 11-17, 2002. 
57. Xing L, Ge C, Zeltser R, Maskevitch G, Mayer BJ and Alexandropoulos $\mathrm{K}$ : c-Src signaling induced by the adapters Sin and Cas is mediated by Rap1 GTPase. Mol Cell Biol 20: 7363-7377, 2000.

58. Martin GS: The hunting of the Src. Nat Rev Mol Cell Biol 2: 467-475, 2001

59. Okutani D, Lodyga M, Han B and Liu M: Src protein tyrosine kinase family and acute inflammatory responses. Am J Physiol Lung Cell Mol Physiol 291: L129-L141, 2006.

60. Tsygankov AY: Non-receptor protein tyrosine kinases. Front Biosci 8: s595-s635, 2003.

61. Williams JC, Weijland A, Gonfloni S, Thompson A, Courtneidge SA, Superti-Furga G and Wierenga RK: The 2.35 A crystal structure of the inactivated form of chicken Src: A dynamic molecule with multiple regulatory interactions. J Mol Biol 274: 757-775, 1997.

62. Okada M, Nada S, Yamanashi Y, Yamamoto T and Nakagawa $\mathrm{H}$ : CSK: A protein-tyrosine kinase involved in regulation of src family kinases. J Biol Chem 266: 24249-24252, 1991.

63. Gregorieff A, Cloutier JF and Veillette A: Sequence requirements for association of protein-tyrosine phosphatase PEP with the Src homology 3 domain of inhibitory tyrosine protein kinase p50 csk. J Biol Chem 273: 13217-13222, 1998.

64. Bouton AH, Riggins RB and Bruce-Staskal PJ: Functions of the adapter protein Cas: Signal convergence and the determination of cellular responses. Oncogene 20: 6448-6458, 2001.

65. Alexandropoulos K and Baltimore D: Coordinate activation of c-Src by SH3-and SH2-binding sites on a novel p130Cas-related protein, Sin. Genes Dev 10: 1341-1355, 1996.

66. Nakamoto T, Sakai R, Ozawa K, Yazaki Y and Hirai H: Direct binding of C-terminal region of p130 to $\mathrm{SH} 2$ and $\mathrm{SH} 3$ domains of Src kinase. J Biol Chem 271: 8959-8965, 1996.
67. Shiozaki A, Shen-Tu G, Bai X, Iitaka D, De Falco V, Santoro M, Keshavjee S and Liu M: XB130 mediates cancer cell proliferation and survival through multiple signaling events downstream of Akt. PloS One 7: e43646, 2012.

68. Viglietto G, Motti ML, Bruni P, Melillo RM, D'Alessio A, Califano D, Vinci F, Chiappetta G, Tsichlis P, Bellacosa A, et al: Cytoplasmic relocalization and inhibition of the cyclin-dependent kinase inhibitor p27(Kip1) by PKB/Akt-mediated phosphorylation in breast cancer. Nat Med 8: 1136-1144, 2002.

69. Zhou BP, Liao Y, Xia W, Spohn B, Lee MH and Hung MC Cytoplasmic localization of p21Cip1/WAF1 by Akt-induced phosphorylation in HER-2/neu-overexpressing cells. Nat Cell Biol 3: 245-252, 2001.

70. Luo J, Manning BD and Cantley LC: Targeting the PI3K-Akt pathway in human cancer: Rationale and promise. Cancer Cell 4: 257-262, 2003.

71. Vivanco I and Sawyers CL: The phosphatidylinositol 3-kinase-AKT pathway in human cancer. Nat Rev Cancer 2: 489-501, 2002.

72. Yamanaka D, Akama T, Fukushima T, Nedachi T, Kawasaki C, Chida K, Minami S, Suzuki K, Hakuno F and Takahashi S: Phosphatidylinositol 3-kinase-binding protein, PI3KAP/XB130, is required for cAMP-induced amplification of IGF mitogenic activity in FRTL-5 thyroid cells. Mol Endocrinol 26: 1043-1055, 2012.

73. Zhao J, Bai X, Wang Y, Keshavjee S and Liu M: Potential role of $\mathrm{XB130}$ in the regulation of airway epithelium repair and regeneration after transplantation. The Journal of Heart and Lung Transplantation 32: S296, 2013. 